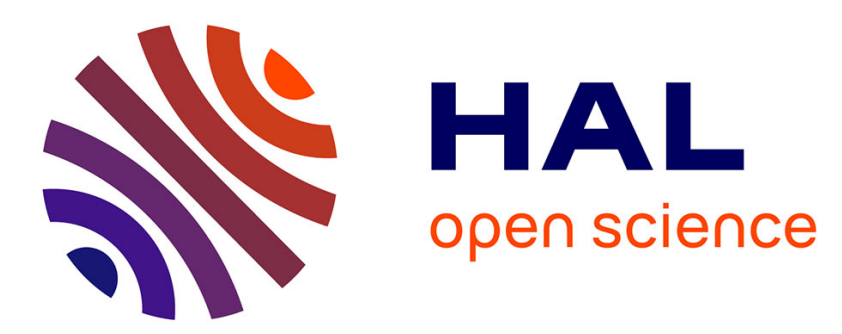

\title{
Intrinsic decoherence and Rabi oscillation damping of Mn 2+and Co 2+ electron spin qubits in bulk $\mathrm{ZnO}$
}

Khalif Benzid, A. Chetoui, M. Maamache, Philippe Turek, Jérôme Tribollet

\section{To cite this version:}

Khalif Benzid, A. Chetoui, M. Maamache, Philippe Turek, Jérôme Tribollet. Intrinsic decoherence and Rabi oscillation damping of Mn 2+and Co 2+ electron spin qubits in bulk ZnO. EPL - Europhysics Letters, 2013, 104 (4), pp.47005. 10.1209/0295-5075/104/47005 . hal-02928869

\section{HAL Id: hal-02928869 https://hal.science/hal-02928869}

Submitted on 2 Sep 2020

HAL is a multi-disciplinary open access archive for the deposit and dissemination of scientific research documents, whether they are published or not. The documents may come from teaching and research institutions in France or abroad, or from public or private research centers.
L'archive ouverte pluridisciplinaire $\mathbf{H A L}$, est destinée au dépôt et à la diffusion de documents scientifiques de niveau recherche, publiés ou non, émanant des établissements d'enseignement et de recherche français ou étrangers, des laboratoires publics ou privés. 


\title{
Intrinsic decoherence and Rabi oscillation damping of $\mathrm{Mn}^{2+}$ and $\mathrm{Co}^{2+}$ electron spin qubits in bulk $\mathrm{ZnO}$
}

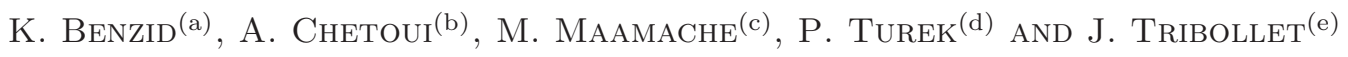 \\ 1/ Institut de Chimie (UMR 7177 CNRS-UDS), Université de Strasbourg, \\ 4 rue Blaise pascal, CS 90032, 67081 Strasbourg cedex, France
}

2/ Département de physique, Laboratoire de Physique Quantique et Systèmes Dynamiques, Université de Ferhat Abbas Sétif 1, Algérie

$\begin{array}{ll}\text { PACS } & 76.30 .-\mathrm{v}- \\ \text { PACS } & 03.67 . \mathrm{Lx}- \\ \text { PACS } & 76.30 . \mathrm{FC}-\end{array}$

\begin{abstract}
We demonstrate by pulse EPR that two electron spin qubits in bulk $\mathrm{ZnO}$, the $\mathrm{Mn}^{2+}$ and the $\mathrm{Co}^{2+}$ spin qubits, which have respectively long $\left(T_{2}(6 K)=178 \mu s\right)$ and short $\left(T_{2}(1.7 \mathrm{~K})=9 \mu \mathrm{s}\right)$ transverse spin coherence time $T_{2}$ at low temperature, have however very short and similar Rabi oscillation damping times, on the order of $T_{R} \approx 250 \mathrm{~ns}$ at low temperature. A detailed study of $\mathrm{Mn}^{2+}$ spin qubits has shown that the main contribution to the Rabi oscilation damping rate is temperature independent and proportional to the Rabi frequency. This main contribution to the damping rate during coherent microwave manipulation of spins is interpreted as due to the changes of the dipolar couplings induced by the long microwave pulse used in this kind of EPR nutation experiment. Strategies are suggested for overcoming this problem of Rabi oscillation overdamping in future spin based quantum computers.
\end{abstract}

Since the first pulse EPR study of a transition metal spin qubit in bulk Zinc Oxide [1], the so called $\mathrm{Fe}^{3+}$ transition metal spin qubit $\left(S=\frac{5}{2}\right)$, showing that intrinsic millisecond spin coherence time $\left(T_{2}\right)$ at helium temperature is possible for some electron spins in the $\mathrm{ZnO}$ matrix, other pulse EPR [2,3] and theoretical [4] studies have been carried out on solid state spin qubits in $\mathrm{ZnO}$. It was shown that inserting the $\mathrm{Mn}^{2+}$ spin qubit $\left(S=\frac{5}{2}\right)$ within a $\mathrm{ZnO}$ nanocrystal with a diameter of only few nanometers and whose surface has been capped by ligands, leads to an unusual and very efficient source of spin decoherence [2]. It was shown that this new source of decoherence is the dipolar couplings between the electron spin $S=\frac{5}{2}$ of the $\mathrm{Mn}^{2+}$ ion and the many nuclear spins of the protons contained in the ligands on the $\mathrm{ZnO}$ nanocrystal surface. This process is not relevant in bulk $\mathrm{ZnO}$, where only very few ${ }^{67} \mathrm{Zn}$ nuclear

\footnotetext{
(a) 1-2/ benzid@unistra.fr

(b) $1-2 /$ chetoui@unistra.fr

(c) 2/ maamache_m@yahoo.fr

(d) $1 /$ turek@unistra.fr

(e) $1 /$ tribollet@unistra.fr : corresponding author
}

spins ( $\frac{4.1}{100}$ natural abundance) of the $\mathrm{ZnO}$ matrix may get involved. We may therefore conclude that bulk $\mathrm{ZnO}$ is more attractive than capped $\mathrm{ZnO}$ nanocrystals for quantum information processing. The use of $\mathrm{Fe}^{3+}$ and $\mathrm{Mn}^{2+}$ as spin qubits in bulk $\mathrm{ZnO}$ was recently highlighted according to the piezoelectric properties of the matrix [3]. The $\mathrm{ZnO}$ piezoelectricity allows manipulating the zero field splitting (ZFS) hamiltonian of any transition metal spin qubit in $\mathrm{ZnO}$ having a spin $S>\frac{1}{2}$ with $\mathrm{AC}$ and $\mathrm{DC}$ electric fields, which can be produced at the nanoscale by metal gates nearby the spins. It thus appears that bulk $\mathrm{ZnO}$ is an excellent host matrix for many electron spin qubits, and particularly for transition metal spin qubits. $\mathrm{ZnO}$ offers the advantages of a solid matrix that can be isotopically purified [4], with strong excitonic optical properties useful as a potential ressource for spin-spin coupling, optical readout and optical initialization of spin qubits [1], and with attractive piezoelectric properties for nanoscale electrical individual manipulations of spin qubits with large spins $S>\frac{1}{2}[3]$.

In this paper, we present a detailed pulse EPR study of the spin coherence properties of ensembles of $\mathrm{Mn}^{2+}$ 
and $\mathrm{Co}^{2+}$ spin qubits in bulk $\mathrm{ZnO}$. This work includes the measurement at $\mathrm{X}$ band of most of the relevant spin hamiltonian parameters of those two qubits through field sweep EPR spectrum, the study of the intrinsic longitudinal spin relaxation time $\left(T_{1}(T)\right)$ and the intrinsic transverse spin relaxation time $\left(T_{2}(T)\right)$ of the qubits as a function of the temperature $\mathrm{T}$ in the range $[1.7 \mathrm{~K}, 40 \mathrm{~K}$, and also the study of the Rabi oscillation damping time $\left(T_{R}\left(T, B_{1}\right)\right)$ of the two kinds of qubits as a function of the microwave magnetic field amplitude $B_{1}$ and temperature T. Despite the fact that $T_{2}(T)$ and $T_{1}(T)$ are found very different for $\mathrm{Mn}^{2+}$ and $\mathrm{Co}^{2+}$ spin qubits in bulk $\mathrm{ZnO}$,quite long for $\mathrm{Mn}^{2+}$ as found previously for the similar $\mathrm{Fe}^{3+}$ spin qubit [1], and quite short for $\mathrm{Co}^{2+}$, which was expected due to the huge ZFS of the $\mathrm{Co}^{2+}$ spin qubit [5], the Rabi oscillation damping times $T_{R}$ were found quite similar and short for the $\mathrm{Co}^{2+}$ and $\mathrm{Mn}^{2+}$ qubits at low temperature. It was also found that the Rabi oscillation damping rate $\frac{1}{T_{R}}$ of the $\mathrm{Mn}^{2+}$ spin qubits is temperature independent at low temperature and has a dominant component linearly dependent on the microwave magnetic field amplitude $B_{1}$, that means on the Rabi frequency which characterizes the speed of the coherent manipulation of the spin qubits. It turns out that $T_{R}$ is much smaller than $T_{2}(T)$ and $T_{1}(T)$ for the two solid state spin qubits investigated here in bulk ZnO. After a detailed presentation of the experimental results, we discuss the possible origines of this problem and its impact on futur quantum computer design with solid state electron spin qubits embedded inside a $\mathrm{ZnO}$ crystal or another crystal.

We used a Bruker Elexsys 580 spectrometer to perform both the pulsed and CW EPR measurements at X band presented here. The temperature of the $\mathrm{ZnO}$ sample could be varied between $4 \mathrm{~K}$ and $40 \mathrm{~K}$ using a CF 935 Oxford cryostat. We also performed some pulsed EPR experiments at $1,7 \mathrm{~K}$ by pumping on the liquid helium bath. The sample studied is a single crystal of wurtzite $\mathrm{ZnO}\left(2 \times 4 \times 0.5 \mathrm{~mm}^{3}\right)$ grown by the Bridgman method and containing, among other trace of impurities, some $\mathrm{Mn}^{2+}$ and $\mathrm{Co}^{2+}$ transition metal ions, both substituted to $\mathrm{Zn}^{2+}$ ions in $\mathrm{ZnO}$.

The EPR spectra shown on the top of figure 1 were recorded by field-sweep CW EPR, respectively at $\mathrm{T}=300 \mathrm{~K}$ for the $\mathrm{Mn}^{2+}$ spins (figure $1 \mathrm{a}$ ), and at $\mathrm{T}=4 \mathrm{~K}$ for the $\mathrm{Co}^{2+}$ spins (figure $1 \mathrm{~b}$ ), in both cases with the magnetic field applied along the $\mathrm{c}$ axis of $\mathrm{ZnO}$. The EPR spectra were also recorded as a function of the angle $\theta$ between the applied magnetic field and the $\mathrm{c}$ axis of wurtzite $\mathrm{ZnO}$ and then simulated using the EasySpin software [6] taking into account the Zeeman Hamiltonian $\left(H_{\text {zeeman }}=\mu_{B} \overrightarrow{B_{0}} \bar{g} \vec{S}\right)$ and also the Hamiltonian of axial magnetic anisotropy existing for the two high spin transition metal ions considered here $\left(H_{\text {aniso }}=+D S_{z}^{2}\right.$, with $S>\frac{1}{2}$ and $z$ parallel to the $c$ axis of $\mathrm{ZnO}$ ). More precisely,
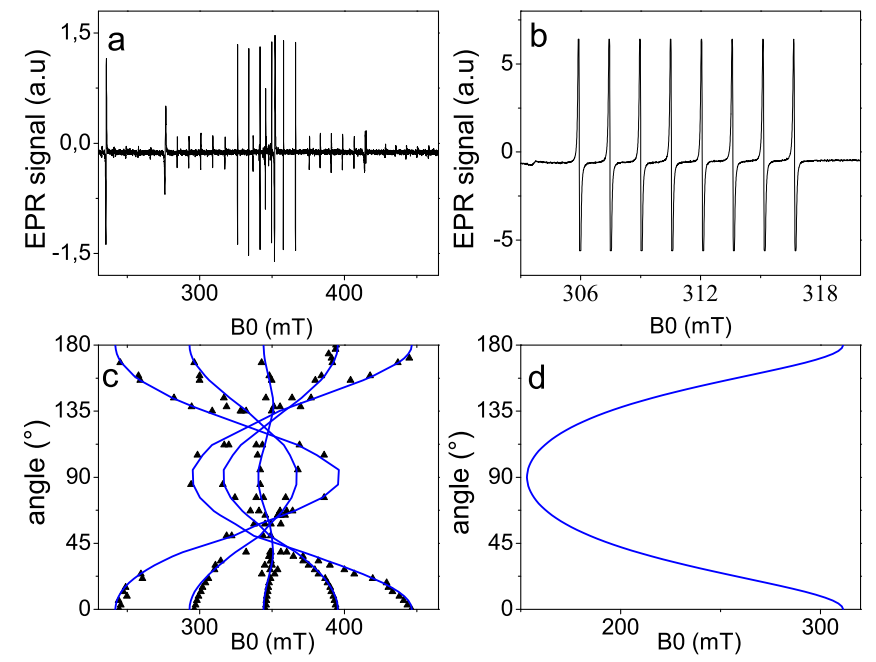

Fig. 1: 1a: field-sweep CW EPR spectrum of $\mathrm{Mn}^{2+}$ spins in $\mathrm{ZnO}$ at $\mathrm{T}=300 \mathrm{~K}$ with magnetic field applied along the $\mathrm{c}$ axis of $\mathrm{ZnO}$; 1b: field-sweep CW EPR spectrum of $\mathrm{Co}^{2+}$ spins in $\mathrm{ZnO}$ at $\mathrm{T}=4 \mathrm{~K}$ with the static magnetic field applied along the c axis of $\mathrm{ZnO}$; 1c: measured (dots) and simulated (continuous lines) rotation pattern of the $\mathrm{Mn}^{2+} \mathrm{EPR}$ spectrum in $\mathrm{ZnO}$ at $\mathrm{T}=300 \mathrm{~K}$ as a function of the angle $\theta ; 1 \mathrm{~d}$ : simulated (continuous line) rotation pattern of the $\mathrm{Co}^{2+} \mathrm{EPR}$ spectrum in $\mathrm{ZnO}$ as a function of the angle $\theta$, obtained using previously measured spin hamiltonian parameters [5] (see also text).

for $\mathrm{Mn}^{2+}$ ions with $S=\frac{5}{2}$ and $I=\frac{5}{2}$ (100/100 abundant), we simulated the measured positions of the five centers of the sets of six EPR hyperfine lines as a function of the angle $\theta$ (figure $1 \mathrm{c}$ ) using an isotropic $\mathrm{g}$ value of $g=2.0029$ and $D=2.3210^{-2} \mathrm{~cm}^{-1}\left(\frac{D}{g \mu_{B}}=24.8 \mathrm{mT}\right.$, it is the absolute value, $\mathrm{D}$ is negative for $\mathrm{Mn}^{2+}$ ions).We also determined the parallel hyperfine coupling for $\mathrm{Mn}^{2+}$ ions, $\frac{A_{/ /}}{g_{\text {iso }} \mu_{B}}=7.57 \mathrm{mT}$ from figure $1 \mathrm{a}$. The hamiltonian parameters we found for $\mathrm{Mn}^{2+}$ ions are in good agreement with previous studies $[7,8]$ (our values corresponds to those of Dorian et al. with less than 3/100 difference). For $\mathrm{Co}^{2+}$ ions with $S=\frac{3}{2}$ and $I=\frac{7}{2}$ (100/100 abundant), we simulated the (not measured) position of the center of the set of eight EPR hyperfine lines associated to the transition $-\frac{1}{2} /+\frac{1}{2}$ using an anisotropic $g$ tensor with $g_{/ /}=2.24$ and $g_{\perp}=4.55$ taken from previous CW EPR study of $\mathrm{Co}^{2+}$ ions in $\mathrm{ZnO}$ as a function of the angle $\theta[5]$. Note that only the transition $-\frac{1}{2} /+\frac{1}{2}$ of $\mathrm{Co}^{2+}$ can be observed at $\mathrm{X}$ band by EPR, due to the large ZFS of the $\mathrm{Co}^{2+}$ ions, previously measured by high field $\mathrm{CW}$ $\operatorname{EPR}\left(D=2.76 \mathrm{~cm}^{-1}\right.$, compared to microwave frequency at $\mathrm{X}$ band: $\left.0.3 \mathrm{~cm}^{-1}\right)[5]$. Thus the $\mathrm{Co}^{2+}$ ions can be viewed as pseudo spins with $s=\frac{1}{2}$ with $I=\frac{7}{2}(100 / 100$ abundant). In this work we found for the $\mathrm{Co}^{2+}$ ions in $\mathrm{ZnO}$ the following hamiltonian parameters $g_{/ /}=2.25$ and $\frac{A_{/ /}}{g_{/ /} \mu_{B}}=1.6 \mathrm{mT}$ (from figure $1 \mathrm{~b}$ ). Those results are also in good agreement with previous studies [5, 9]. 
The various contributions to the spin hamiltonians for both $\mathrm{Co}^{2+}$ and $\mathrm{Mn}^{2+}$ spin qubits being known (Zeeman , quadratic magnetic anisotropy and hyperfine, others being neglected), we could study the relevant intrinsic decoherence processes for those two spin qubits at low temperature, and with the static magnetic field applied along the $\mathrm{c}$ axis of $\mathrm{ZnO}$. According to the previous study of the $\mathrm{Fe}^{3+}$ spin qubits in bulk $\mathrm{ZnO}$ [1], the phase memory time, $T_{m}$, was determined at various temperature for both $\mathrm{Co}^{2+}$ and $\mathrm{Mn}^{2+}$ spins by measuring the two pulses echo decay $\left(\frac{\pi}{2}-\tau-\pi-\tau-e c h o, \tau\right.$ varying). The three pulses inversion recovery experiment $\left(\pi-t_{\text {wait }}-\frac{\pi}{2}-\tau-\pi-\tau-\right.$ echo, $t_{\text {wait }}$ varying ) was also used to determine the spin-lattice relaxation time, $T_{1}$, at several temperature for both $\mathrm{Co}^{2+}$ and $\mathrm{Mn}^{2+}$ spins [1]. The temperature dependence of $\frac{1}{T_{1}}$ and $\frac{1}{T_{m}}$ for $\mathrm{Mn}^{2+}$ spin qubits is shown on figure 2 .
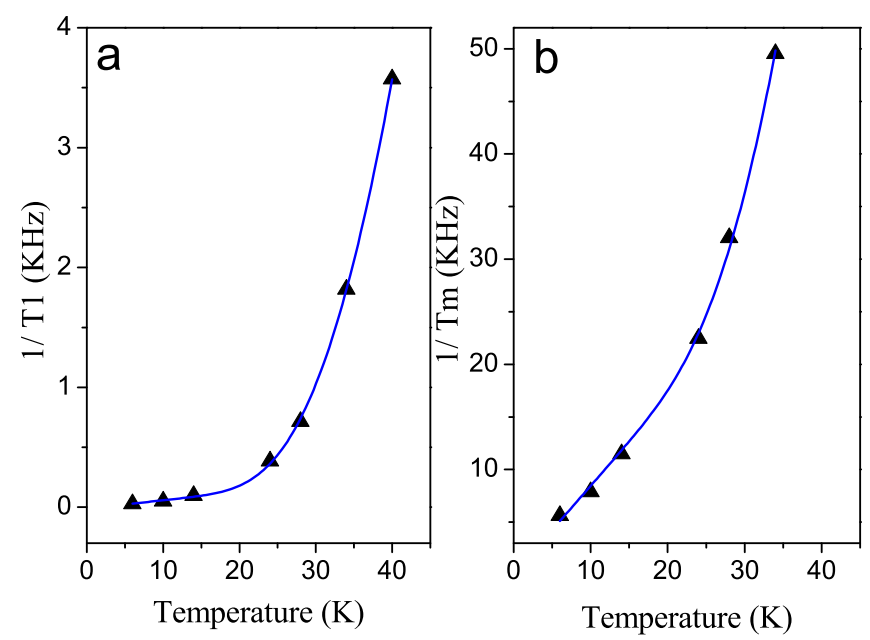

Fig. 2: Temperature dependence of the longitudinal relaxation rate $\frac{1}{T_{1}}(\mathrm{a} /)$, and of the inverse of the phase memory time $\frac{1}{T_{m}}$ (b/), for $\mathrm{Mn}^{2+}$ spins qubits in $\mathrm{ZnO}$ between $6 \mathrm{~K}$ and $40 \mathrm{~K}$. Solid lines are fit as described in the main text.

For example, at $\mathrm{T}=6 \mathrm{~K}$, we have for $\mathrm{Mn}^{2+}$ spins, $T_{2}(6 \mathrm{~K})=178 \mu \mathrm{s}$ and $T_{1}(6 \mathrm{~K})=35 \mathrm{~ms}$ (see figure 2 ), and for $\mathrm{Co}^{2+}$ spins, $T_{2}(1.7 K)=9 \mu \mathrm{s}$ and $T_{1}(1.7 K)=40 \mu \mathrm{s}$ (data not shown). For the $\mathrm{Co}^{2+}$ spin qubit, pulsed EPR measurement above $\mathrm{T}=4 \mathrm{~K}$ were not performed because the spin coherence time $T_{2}(T=4 K) \approx 1.17 \mu \mathrm{s}$ was already quite short. For the $\mathrm{Mn}^{2+}$ spin qubit, extracting $T_{2}(T)$ below $\mathrm{T}=6 \mathrm{~K}$ is difficult because instantaneous diffusion, nuclear spectral diffusion, as well as spectral diffusion due to the spin flips of nearby $\mathrm{Co}^{2+}$ spins, should all be taken into account $[1,10-12]$. This results in an overparametrized problem, leading to a low confidence in the obtained results. Restricting the analysis between $6 \mathrm{~K}$ and $40 \mathrm{~K}$ for $\mathrm{Mn}^{2+}$ spin qubits, and between $1.7 \mathrm{~K}$ and $4 \mathrm{~K}$ for $\mathrm{Co}^{2+}$ spin qubits, one shows below that the dominant spin decoherence processes in those respective temperatures ranges are temperature dependent for both kinds of qubits. Instantaneous diffusion being neglected in this case, the phase memory time $T_{M}$ measured by echo decay is equal to the temperature dependent decoherence time called $T_{2}(T)$ related to spin-lattice relaxation [1,10-12]. The observed similarity between the spin hamiltonian of the $\mathrm{Mn}^{2+}$ spin qubit studied here and the previously studied $\mathrm{Fe}^{3+}$ spin qubit [1] (apart from the slightly different ZFS and the fact that $\mathrm{Mn}^{2+}$ ions have 100/100 abundant nuclear spins $I=\frac{5}{2}$ ), leads us to assume a similar origin for the thermally induced intrinsic decoherence processes of $\mathrm{Mn}^{2+}$ and $\mathrm{Fe}^{3+}$ spins in $\mathrm{ZnO}$ $\left(\frac{1}{T_{j}(T)}=A_{j} T+B_{j} \exp \left(-T_{0 j} / T\right), j=1\right.$ or $\left.m\right)$, a one phonon direct relaxation process at very low temperature and a two optical phonon Raman decoherence process activated at much higher temperature, with an activation energy $k T_{0, j}=(12 \pm 1) \mathrm{meV}, j=1$ or $m$, corresponding to the energy of the transverse optical phonons in bulk $\mathrm{ZnO}[1,13,14]$. The quality of the fitting curves of experimental data shown on figure 2 shows the relevance of the analysis. It must be also noted that both $T_{m}(T)$ and $T_{1}(T)$ found here for $\mathrm{Mn}^{2+}$ spins in bulk $\mathrm{ZnO}$ are much longer than those found previously in small $\mathrm{ZnO}$ nanocrystals capped with protonated ligands which induce a stronger spin decoherence [2]. In the case of the $\mathrm{Co}^{2+}$ spin qubits in bulk $\mathrm{ZnO}$, we found $T_{1}(1.7 \mathrm{~K})=40 \mu \mathrm{s}$ and $T_{1}(4 K)=1.3 \mu \mathrm{s}$. The only two experimental points available did not allow us to perform a reliable analysis of the temperature dependence of $\frac{1}{T_{1}}$. More points would be needed, which was not experimentally feasible with our setup. Nevertheless, the measurements show that $T_{1}$ and $T_{2}$ of $\mathrm{Co}^{2+}$ spin qubits in bulk $\mathrm{ZnO}$ are temperature dependent in this low temperature range.

Given the knowledge of $T_{2}(T)$ of the isolated $\mathrm{Mn}^{2+}$ and $\mathrm{Co}^{2+}$ spin qubits in bulk $\mathrm{ZnO}$, we can in principle evaluate their quality for quantum information processing, considering that the typical time, $T_{\text {gate, necessary to per- }}$ form a single qubit rotation with a microwave pulse is in the range of $10 \mathrm{~ns}$. In that case, the figure of merit $T_{2}(T) / T_{\text {gate }}$ of the $\mathrm{Mn}^{2+}$ spin qubit at $\mathrm{T}=6 \mathrm{~K}$ would be $T_{2}(T=6 K) / T_{\text {gate }}=1.710^{4}$ and the one of $\mathrm{Co}^{2+} \mathrm{spin}$ qubit at $\mathrm{T}=1.7 \mathrm{~K}$ would be $T_{2}(T=1.7 \mathrm{~K}) / T_{\text {gate }}=900$. According to its figure of merit above $10^{4}$ deduced at $6 \mathrm{~K}$, the $\mathrm{Mn}^{2+}$ ion would provide an excellent isolated spin qubit, and even more at much lower temperature, meaning that error correction protocol could be applied to it at a sufficiently fast rate [15]. This is no longer valid for the $\mathrm{Co}^{2+}$ ion due to its faster decoherence rate even at $1.7 \mathrm{~K}$. However, in a relevant quantum computation, many quantum gates are applied as fast as possible to the register of qubits in order to manipulate the quantum information encoded on the entangled spin states. For the useless but simple case of a single qubit quantum register, that means that instead of considering the figure of merit $T_{2}(T) / T_{\text {gate }}$, one should consider the new figure of merit $T_{R}(T) / T_{\text {gate }}$, where $T_{R}(T)$ is the damping time of a spin nutation experiment performed in principle on a single isolated electron spin qubit by pulsed EPR methods [10]. 
This is due to the fact that in such an experiment, the microwave field is continuously applied to the electron spin qubit (except during the echo detection sequence using few short microwave pulses). This is in a first approximation, similar to applying a fast and dense sequence of successive microwave pulses implementing different single qubit quantum gates, as it would be the case in a genuine quantum computation. This has to be compared to the simple Hahn echo decay experiment performed to get the $T_{2}$ damping time of the spin echo. This requieres only two short and well time separated microwave pulses, meaning that most of the time the electron spin is freely evolving without any applied microwave field. However, the experimental problem related to this spin nutation experiment, also called a Rabi oscillation experiment in the field of quantum computing, is that it can not in general be performed on a single isolated solid state electron spin qubit by pulse EPR methods [10] due to the lack of sensitivity of standard inductively detected EPR. As a consequence, the isolated qubit figure of merit of most of the EPR studies of electron spin qubits was evaluated by the simple formula $T_{2}(T) / T_{\text {gate }}$ estimated using ensemble EPR measurements. Rabi oscillation experiments were performed in a few cases, but most of the time again on ensemble of electron spin qubits $[2,16]$. This is however a more realistic situation because in a solid state quantum computer, many identical atoms or ions or defects encoding electron spin qubits should be close enough to have some spin-spin inter-qubit coupling in order to build controlled two qubits quantum gates.

The nutation (Rabi oscillation) experiment consists in applying a first microwave nutation pulse of increasing length $t_{n u t}$, followed by a fixed standard spin echo detection sequence, all microwave pulse being in resonance with an EPR transition of the spin qubit [2]. As it can be seen on figure $3 \mathrm{a}, T_{R}(T=10 \mathrm{~K}) \approx 284 \mathrm{~ns}$ for $\mathrm{Mn}^{2+}$ spin qubits, and as shown on figure $3 \mathrm{c}$, $T_{R}(T=1.7 K) \approx 224 \mathrm{~ns}$ for $\mathrm{Co}^{2+}$ spin qubits. It is thus demonstrated that despite the fact that $T_{2}(T)$ and $T_{1}(T)$ are found very different for $\mathrm{Mn}^{2+}$ and $\mathrm{Co}^{2+}$ spin qubits in bulk $\mathrm{ZnO}$, the Rabi oscillation damping times $T_{R}$ were found quite similar and short for the $\mathrm{Co}^{2+}$ and $\mathrm{Mn}^{2+}$ qubits at low temperature in the present bulk $\mathrm{ZnO}$ sample. The nutation damping time of $\mathrm{Mn}^{2+}$ spins was found constant between $\mathrm{T}=6 \mathrm{~K}$ and $\mathrm{T}=14 \mathrm{~K}$ (results not shown). Varying the amplitude of the oscillating magnetic field corresponds to varying the Rabi frequency. The Rabi oscillation damping rates of the $\mathrm{Mn}^{2+}$ transition metal spin qubits in bulk $\mathrm{ZnO}$ were thus recorded as a function of the Rabi frequency (inset b/ of figure 3). A linear dependence is obvious as previously demonstrated with other solid state spin qubits in different matrix $[2,16]$. Previous studies have shown an interesting concentration dependence of the Rabi damping time in some paramagnetic solid material [16]. The concentration of $\mathrm{Mn}^{2+}$ transition metal spin qubits was estimated in our sample by performing a Hahn echo decay experiment
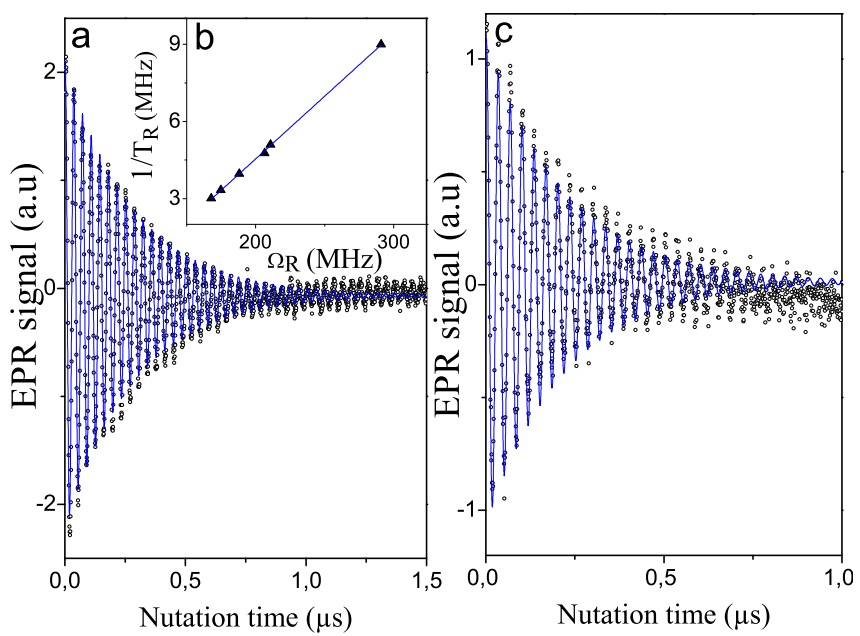

Fig. 3: a/ Damped Rabi oscillations measured over an ensemble of $\mathrm{Mn}^{2+}$ spin qubits at $\mathrm{T}=10 \mathrm{~K}$ with a static magnetic field parallel to the $\mathrm{c}$ axis of $\mathrm{ZnO}$ (Bres= $328 \mathrm{mT}$ ). b/ inset: dependence of the Rabi oscillation decay rate $\frac{1}{T_{R}}$ on Rabi frequency $\Omega_{R}$ for $\mathrm{Mn}^{2+}$ spin qubits at $\mathrm{T}=10 \mathrm{~K}$. The fitting formula used was of the form: $\frac{1}{T_{R}}=A+B \Omega_{R}$, with $\mathrm{A}$ and $\mathrm{B}$ two constants. c/ Damped Rabi oscillations measured over an ensemble of $\mathrm{Co}^{2+}$ spin qubits at $\mathrm{T}=1.7 \mathrm{~K}$ with a static magnetic field parallel to the $\mathrm{c}$ axis of $\mathrm{ZnO}$ (Bres $=311.4 \mathrm{mT}$ ). The fit of the curves on figures $3 \mathrm{a} /$ and $3 \mathrm{c} /$ are the solid lines.

at $\mathrm{T}=4 \mathrm{~K}$ with a large number of averaging cycles and sufficient time delay increment steps, thus evidencing small oscillations superimposed on the echo decay curve (figure 4 a) [10]. Once background corrected (figure $4 \mathrm{~b})$, the Fourier transform of these oscillations shows three maxima(figure $4 \mathrm{c}$ ). The main one occurs at 0.04 $\mathrm{MHz}$, which is too low to be assigned to a known nuclear spin precession frequency at the resonant magnetic field used in this experiment. This excludes the possibility of a standard nuclear ESEEM effect [10]. Another reasonable explanation is that this oscillation reflects a quite weak magnetic coupling energy, e.g. the dipolar magnetic coupling between two on resonance $\mathrm{Mn}^{2+}$ spins separated by a large distance d. Following that way [10], one estimates $d=10.8 \mathrm{~nm}$, which corresponds to an effective concentration $C_{\text {eff }} \approx 7.10^{17} \mathrm{~cm}^{-3}$. As already mentioned, previous pulse EPR studies in another paramagnetic solid have shown that the Rabi oscillation damping time was concentration dependent [16] within this range of concentration. This suggests that it is also probably the case in the studied $\mathrm{ZnO}$ sample in this range of $\mathrm{Mn}^{2+}$ spins concentration.

The physical origin of this unwanted additional Rabi oscillation damping rate is still under debate. It could be due to some inhomogeneity of the microwave magnetic field amplitude within the volume of the sample [2]. Such inhomogeneity would be intrinsic to the spectrometer experimental setup. Some fluctuations of the total microwave magnetic field probed by each single 
spin within the spins ensemble could also occur under continuous wave irradiation. Accordingly, fluctuations
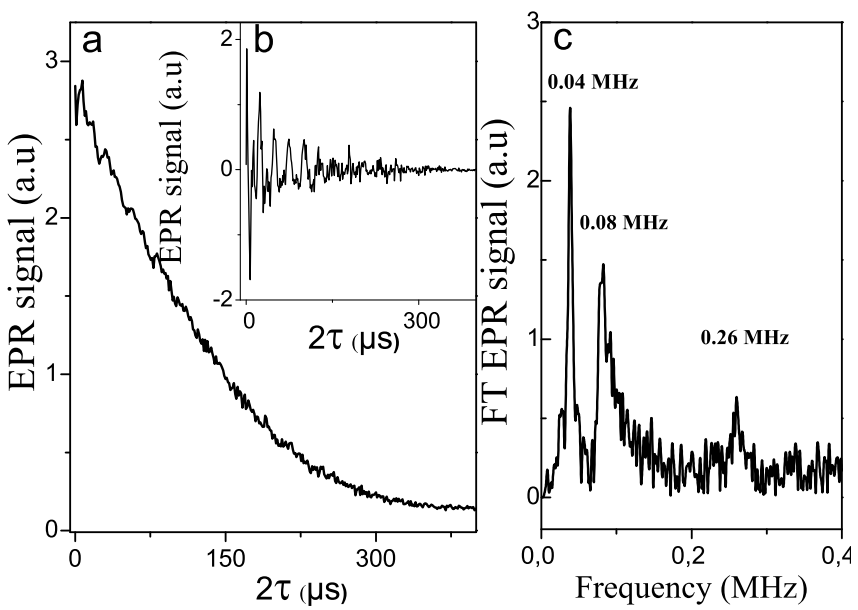

Fig. 4: a/ Echo decay curve of $\mathrm{Mn}^{2+}$ spins in $\mathrm{ZnO}$ recorded at $\mathrm{T}=4 \mathrm{~K}$ and modulated by electron-electron dipolar couplings. b/ Same data as in a/, but after background decay substraction. c/ Fourier transform of the curve shown in b/.

of the microwave source itself were recently excluded by some test experiments [16], but it was also suggested that this additional Rabi oscillation damping rate could be due to the dipolar coupling changes induced by the continuous wave irradiation of the whole sample [16]. This hypothesis is based on a recent theory [17] describing this effect and on the experimental observation that it depends on the spin concentration in the sample [16]. Whatever its microscopic origin, it seems more and more obvious from recent experiments $[2,16]$ and theory [17], that this additional damping rate of Rabi oscillations is always proprotional to the Rabi frequency and to the spin concentration. This has important consequences on the design of future solid state based electron spins quantum computer, which also depends on the strategy used to coherently manipulate the electron spins inside the quantum register. In a first strategy, the most common one, one would perform ensemble EPR coherent manipulations and measurements on many copies of the same one dimensional chain of coupled electron spins qubits. In that case, the microwave magnetic field would be applied globally on the whole sample which means simultaneously on all copies of a same qubit. In order to keep long spin coherence time for coherent manipulation this implies that the many one dimensional chains of electron spins qubits would have to be well separated laterally to avoid the fast damping of Rabi oscillations that would occur for closely spaced chains of spin qubits. The final consequence of this first strategy is that one would certainly need to increase the sensitivity of the EPR signal detection, due to the necessary dilution of the chains of spin qubits inside the solid sample. A possible solution in that case would be to use a highly sensitive method for the detection of the electron spin dynamic, e.g. as provided by optical detection [18-20]. In a second strategy, the less common one, one would be able to perform coherent manipulations on each single electron spin qubit inside a single chain of electron spin qubits used as the solid state quantum register. In that case, the single spin coherent manipulation would be possible if one is able to apply an electromagnetic field confined at the nanometer scale relevant to the spacing between a qubit and the next one in the register. This strategy is generally proposed for electron spin qubits, which can be efficiently coherently manipulated by local AC electric field [21]. However, such electrical manipulation is restricted to magnetic systems with a large enough spin-orbit coupling [21]. This has the disadvantage that it makes those particular qubits also more sensitive to decoherence processes induced by random charge fluctuation. However, for transition metal spin qubits in $\mathrm{ZnO}$ the situation is different and that key point must be underlined. As a matter of fact the recently demonstrated electrical manipulation of the $\mathrm{Mn}^{2+}$ spin qubits in $\mathrm{ZnO}$ [3] was possible according to the good piezoelectric properties of the bulk $\mathrm{ZnO}$ matrix. The piezoelectric effect allows modulating the local strain field on the spin qubit by the applied electric field, AC or DC, and subsequently, its zero field splitting, which depends on the local crystal field. Thus, one can see that some transition metal spin qubits in bulk $\mathrm{ZnO}$, like the previously studied $\mathrm{Fe}^{3+}$ spin qubits [1] or the $\mathrm{Mn}^{2+}$ spin qubits here studied, could offer a simple alternative to solve the present problem of the additional damping rate of Rabi oscillations. Assuming that the state-of-the-art nanotechnology could reliably build up arrays of nanoscale metal electrodes on a bulk $\mathrm{ZnO}$ surface, the individual coherent manipulation of a single spin by a local AC electric field would not affect, in principle, the neighboring electron spin qubits. One can note that even with this strategy, the individual coherent manipulation of a single spin would probably also imply the need for a single spin detection of the EPR signal of the qubit, which here again requires an highly enhanced sensitivity of the EPR signal detection setup.

In conclusion, we have here demonstrated by pulse EPR spectroscopy that two electron spin qubits in bulk $\mathrm{ZnO}$, the $\mathrm{Mn}^{2+}$ spin qubits and the $\mathrm{Co}^{2+}$ spin qubits, while having respectively very long and very short transverse spin coherence time at cryogenic temperatures, have however very short and similar Rabi oscillation damping times on the order of three hundreds of nanoseconds at low temperature. A detailed study performed on $\mathrm{Mn}^{2+}$ spin qubits has shown that the Rabi oscillation damping rate has a main contribution which is temperature independent and proportional to the Rabi frequency at low temperature. The $\mathrm{Mn}^{2+}$ spin qubits concentration estimated by pulse EPR in the studied bulk $\mathrm{ZnO}$ sample $\left(\approx 7.10^{17} \mathrm{~cm}^{-3}\right)$, appears large enough to interpret the main contribution 
of the Rabi oscillation damping rate of $\mathrm{Mn}^{2+}$ spin qubits as being due to the changes of the dipolar coupling induced by the long microwave pulse used in this kind of EPR nutation experiment, in good agreement with recent experiments and theories. To overcome this problem of Rabi oscillation overdamping in future quantum computers, either a dilution of the copies of the quantum registers in the solid state device will be necessary or an individual coherent manipulation of each single qubit of the register using a local AC electric field applied to each qubit. This last solution should be possible with the previously studied $\mathrm{Fe}^{3+}$ spin qubits and also with the $\mathrm{Mn}^{2+}$ spin qubits studied here, due to the excellent piezoelectric properties of bulk $\mathrm{ZnO}$.

\section{REFERENCES}

[1] J. Tribollet et al., Europhysics Letters 84, 20009 (2008).

[2] S.T. Ochsenbein et al., Nature Nanotechnology 6, 112 (2011).

[3] R.E. George et al., Phys. Rev. Lett. 110, 27601 (2013).

[4] J. Tribollet, Eur. Phys. J. B 72, 531 (2009).

[5] P. Sati et al., Phys. Rev. Lett. 96, 17203 (2006).

[6] S. Stoll and A. Schweiger, J. Magn. Res. 178, 42 (2006).

[7] P.B. Dorain, Phys. Rev. 112, 1058 (1958).

[8] A. Haussman et al., J. Phys. Chem. Sol. 29, 1369 (1968).

[9] A. Haussman, Phys. Stat. Sol. b 31, K131 (1969).

[10] A. Schweiger and G. Jeschke, Principles of pulse electron paramagnetic resonance (Oxford University Press, Oxford, UK ; New York, 2001).

[11] C. Cohen-Tannoudji and J. Dupont-Roc, Processus d'interaction entre photons et atomes (EDP Sciences, 1996).

[12] D. Gamliel and H. Levanon, Stochastic processes in magnetic resonance (World Scientific, 1995).

[13] A. Abragam and B. Bleaney, Electron paramagnetic resonance of transition ions (Clarendon Press, Oxford, 1970).

[14] C. Y. Huang, Phys. Rev. 154, 215 (1967).

[15] D. P. DiVincenzo, Fortschr. d. Phys. 48, 771 (2000).

[16] S. Agnello et al., Phys. Rev. A 59, 4087 (1999).

[17] R.N. Shakhmuratov et al., Phys. Rev. Lett. 79, 2963 (1997).

[18] L. Besombes et al., Phys. Rev. Lett. 93, 207403 (2004).

[19] J. Tribollet et al., Phys. Rev. B 75, 205304 (2007).

[20] P.G. Baranov et al., JETP Letters 84, 400 (2006).

[21] V.N. Golovach et al., Phys. Rev. B 74, 165319 (2006). 